Clinical Papers

\title{
A new methodology for assessment of pectus excavatum correction after bar removal in Nuss procedure: Preliminary study
}

\author{
João Gomes-Fonseca ${ }^{\text {a,b,*,1 }}$, João L. Vilaça ${ }^{\text {a,b,c,1 }}$, Tiago Henriques-Coelho ${ }^{\text {d,e }}$, Bruno Direito-Santos ${ }^{\text {a,b,f }}$, \\ António C.M. Pinho ${ }^{g}$, Jaime C. Fonseca ${ }^{h}$, Jorge Correia-Pinto ${ }^{\mathrm{a}, \mathrm{b}, \mathrm{i}}$
}

\footnotetext{
a Life and Health Sciences Research Institute (ICVS), School of Medicine, University of Minho, Braga, Portugal

b ICVS/3B's-PT, Government Associate Laboratory, Braga/Guimarães, Portugal

c DIGARC-Technology School, Polytechnic Institute of Cávado and Ave, Barcelos, Portugal

d Department of Pediatric Surgery, Centro Hospitalar de São João, Porto, Portugal

e Department of Pediatrics, Faculty of Medicine, University of Porto, Porto, Portugal

${ }^{\mathrm{f}}$ Department of Orthopedics, Hospital de Braga, Braga, Portugal

${ }^{g}$ Department of Mechanical Engineering, School of Engineering, University of Minho, Guimarães, Portugal

${ }^{\mathrm{h}}$ Department of Industrial Electronics, School of Engineering, University of Minho, Guimarães, Portugal

i Department of Pediatric Surgery, Hospital de Braga, Braga, Portugal
}

\section{A R T I C L E I N F O}

\section{Article history:}

Received 7 March 2016

Received in revised form 30 December 2016

Accepted 31 December 2016

\section{Key words:}

Nuss procedure

Pectus excavatum

Postretraction

3D surface analysis

3D surface scanning

\begin{abstract}
A B S T R A C T
Purpose: The objective is to present a new methodology to assess quantitatively the impact of bar removal on the anterior chest wall, among patients with pectus excavatum who have undergone the Nuss procedure, and present a preliminary study using this methodology.

Methods: We propose to acquire, for each patient, the surface of the anterior chest wall using a three-dimensional laser scanner at subsequent time points (short term: before and after surgery; long term: follow-up visit, 6 months, and 12 months after surgery). After surfaces postprocessing, the changes are assessed by overlapping and measuring the distances between surfaces.

In this preliminary study, three time points were acquired and two assessments were performed: before $v s$ after bar removal (early) and before vs 2-8 weeks after bar removal (interim). In 21 patients, the signed distances and volumes between surfaces were computed and the data analysis was performed.

Results: This methodology revealed useful for monitoring changes in the anterior chest wall. On average, the mean, maximum, and volume variations, in the early assessment, were $-0.1 \pm 0.1 \mathrm{~cm},-0.6 \pm 0.2 \mathrm{~cm}$, and $47.8 \pm 22.2 \mathrm{~cm}^{3}$, respectively; and, in the interim assessment, were $-0.5 \pm 0.2 \mathrm{~cm},-1.3 \pm 0.4 \mathrm{~cm}$, and $122.1 \pm 47.3 \mathrm{~cm}^{3}$, respectively $(p<0.05)$. Data analysis revealed that the time the bar was in situ was inversely and significantly correlated with postretraction and was a relevant predictor of its decrease following surgery $(p<0.05)$. Additionally, gender and age suggested influencing the outcome.

Conclusions: This methodology is novel, objective and safe, helping on follow-up of pectus excavatum patients. Moreover, the preliminary study suggests that the time the bar was in situ may be the main determinant of the anterior chest wall retraction following bar removal. Further studies should continue to corroborate and reinforce the preliminary findings, by increasing the sample size and performing long-term assessments.

Levels of evidence: III
\end{abstract}

(c) 2017 Elsevier Inc. All rights reserved.
Pectus excavatum (PE) is the most common congenital chest wall abnormality and it is characterized by both sternal and costal cartilage depression [1]. The estimated occurrence is 1 in 400 to 1 in 1000 live births and it affects more frequently male than female patients at a ratio of $3-5: 1[1,2]$.

\footnotetext{
* Corresponding author at: Life and Health Sciences Research Institute (ICVS), School of Medicine, University of Minho, Campus de Gualtar, 4710-057 Braga, Portugal. Tel.: + 351 253604 891; fax: + 351253604847

E-mail address: joaofonseca@med.uminho.pt (J. Gomes-Fonseca).

${ }^{1}$ These authors contributed equally to this work.
}

The Nuss and Ravitch procedures are well-established surgical techniques to correct PE. Meta-analysis suggests good outcomes from both and slightly better outcomes in pediatric Nuss procedure patients [3]. Nuss surgical technique involves the insertion of a prebent metal bar through two axillary incisions in the retrosternal space. The bar remains in situ for at least two years $[1,4]$.

Despite the successful results of the Nuss procedure [4-7], some authors showed that retraction of the anterior chest wall (ACW) following bar removal can occur [8-10]. However, they evaluated and presented this hypothesis using approaches without advanced technology. In fact, some authors have been researching the use of three- 
dimensional (3D) laser scanners to study pectus deformities [11,12] and to improve their treatment [13]. Given advances in imaging tools, assessment of the ACW should rely on objective measurements to document postretraction in terms of degree, timing, and influencing factors. In this sense, the present study aims to introduce a new methodology to assess changes in the ACW morphology, over time, after pectus bar removal.

\section{Materials and methods}

In this section, we describe a new methodology to assess changes after pectus bar removal, based on 3D surface topography data, and then, we present a preliminary study using this methodology. For further technical and mathematical details of the proposed methodology, consult Appendix A.

\subsection{New methodology}

\subsubsection{System}

We propose to acquire the ACW surfaces using a handheld 3D laser scanner, the Polhemus FastSCAN ${ }^{\mathrm{TM}}$ Cobra (Polhemus, Colchester, VT). This system has a wand with a laser light and a camera, which records the cross-sectional depth profiles of the objects, and an electromagnetic motion tracking system, which determines the position and orientation of the wand (Fig. 1-A). As well, this system has a processor unit that computes and transfers the 3D data to the computer in real time (data are stored and displayed on the monitor as triangular meshes). This highresolution system (maximum resolution: $0.01 \mathrm{~cm}$ ) allows portability, quick and comfortable acquisitions of the entire surface of the ACW (less than 1-2 min), real-time perception and correction of errors, and avoids radiation and cost of multiple exams such as computed tomography scan (CT scan). Altogether, these characteristics benefit those involved.

\subsubsection{ACW surfaces acquisition and postprocessing}

During acquisition, the patient must remain in dorsal decubitus position with their arms away from the thorax and parallel to both transverse and coronal planes. This patient positioning is comfortable and allows a complete access to the ACW. During acquisition, the operator must ask the patient to hold breathing for short periods, as is the practice in a normal chest CT scan. The wand-to-body range must be kept up to $20 \mathrm{~cm}$ far from the patient's body to keep up the resolution along the laser line in 0.01 to $0.05 \mathrm{~cm}$.

Because of the electromagnetic motion tracking system, the scanner requires a reference/transmitter. We use the Short Ranger reference (Polhemus, Colchester, VT) with a volume radius of the electromagnetic field up to $30 \mathrm{~cm}$ and position it under the fifth or the sixth rib and away from the PE's correction area (Fig. 1-A/B). Thereby, we intend to make the setup simpler and faster, comprise the full ACW inside the magnetic tracking volume (narrowing the errors), and, at the same time, avoid influencing the surface shape itself.

Given the influence of the scanner, the operator, and the environment during the acquisition, each ACW surface should be acquired in several sweeps and these sweeps must overlap each other (Fig. 1-B). This procedure allows postcorrection of small misalignments and slight body movements between sweeps. The number of sweeps depends on the operator and the ACW shape, and sweeps should represent the entire ACW. Then, each surface must be reconstructed with a high accuracy (reducing noise and improving surface quality), using the tools within Polhemus FastSCAN software (version 4.0.7) to select, align, merge, filter (Fig. 1-C), and improve smoothness and eliminate holes (Fig. 1-D) using each one of the sweeps available.

As an additional step, we suggest acquiring the ACW surface at least 2 times for each time point. Then, ACW surfaces must be merged and an average surface must be created using a surface reconstruction algorithm, namely screened Poisson surface reconstruction [14]. With this step, we intend to mitigate, even more, the acquisition errors.

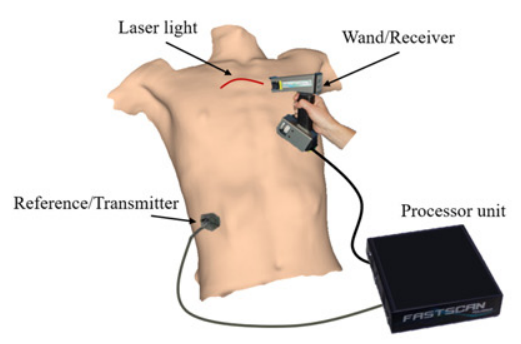

A

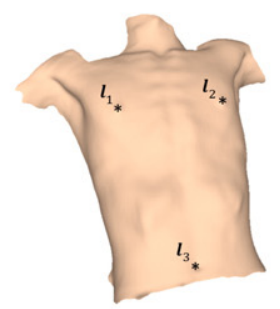

E

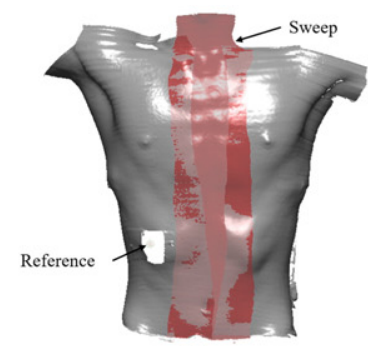

B

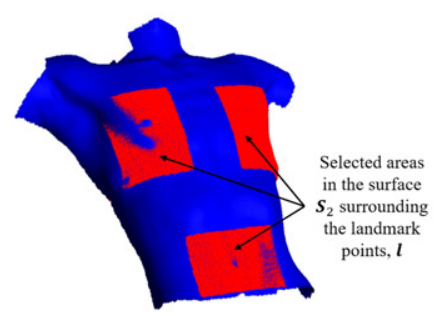

F

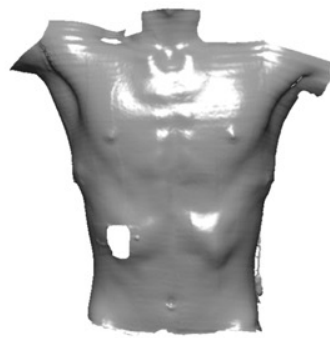

C

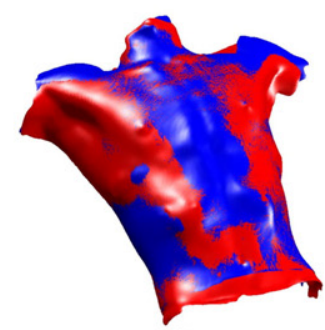

G

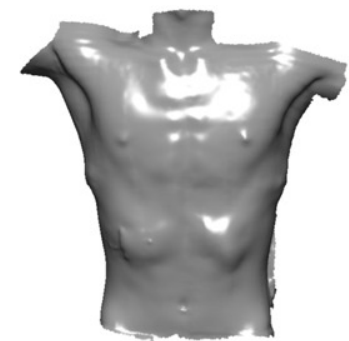

D

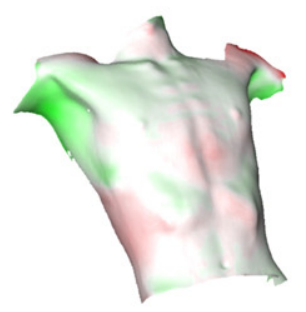

$\mathrm{H}$

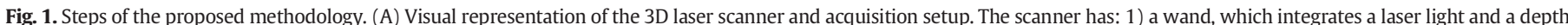

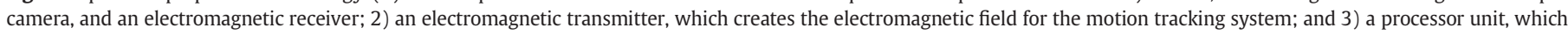

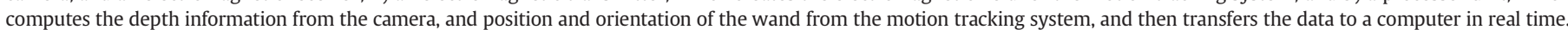

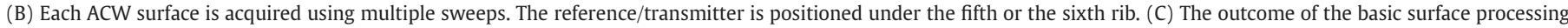

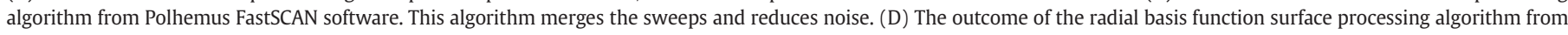

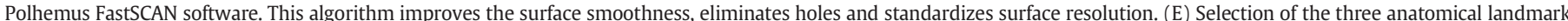

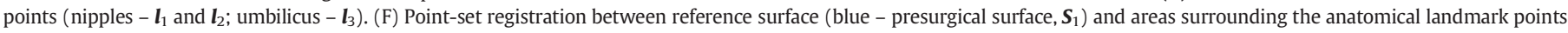

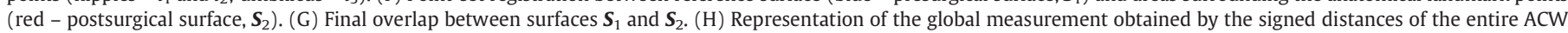
surface (red - retraction; green - protrusion). 


\subsubsection{ACW surfaces assessment}

Because of the lack of quantitative studies related to PE correction, we propose to study the ACW evolution by overlapping the acquired surfaces and quantifying the differences at subsequent time points for each patient, e.g. surfaces acquired before and after surgery. Similarly, these studies should be extended to long-term acquisitions, as a follow-up after surgery, 6 months after surgery, and 12 months after surgery, among others. To this end, surfaces assessment must include an alignment step and then a measurement step.

Overlapping surfaces can be performed manually, automatically or through both. Manual alignment is time consuming and userdependent, and the alignment error is generally high, if landmark points do not correspond to each other. Automatic alignment requires good initializations (if both surfaces are not initially close, it can lead to wrong solutions) and computational time, and the alignment error is generally low because methods automatically minimize the distances between two surfaces. Therefore, a valid option is combining both strategies. For initialization, we propose to begin the alignment step by manually selecting three correspondent anatomical landmarks (Fig. 1-E, umbilicus, and nipples) on both ACW surfaces. These selected landmarks are used to approximate the surfaces by computing the rigid transformation based on the singular value decomposition method [15]. Next, to refine the alignment, a point-set registration method, the iterative closest point (ICP) which minimizes the distances between points [16], is applied using more points of the ACW surfaces. Yet, we do not suggest using all points of the surfaces. Instead, to not take into account variations introduced by bar removal and small misalignments (e.g. small body displacements, dressings and swelling) in the pointset registration, which will lead to incorrect measurements, we suggest using a local approach. The main idea is to automatically limit and find the areas where less variations occurred between two consecutive surfaces obtaining the most coherent alignment. Thus, in multiple iterations, areas with different sizes surrounding the anatomical landmarks (Fig. 1-F) must be automatically changed, and then, using an optimization method $[17,18]$, the best ICP alignment is automatically selected (Fig. 1-G).

After the alignment, the measurement step is undertaken to quantify changes between surfaces. These changes can be largely explored through the 3D data. One can obtain global and localized measurements by computing distances (Fig. 1-H) between surfaces under assessment. As above mentioned, these surfaces are represented as triangular meshes, which are represented by a set of points (or vertices) in $\mathbb{R}^{3}$, a set of edges and a set of triangles (or faces) describing how the vertices are linked together. We define two consecutive surfaces as $\boldsymbol{S}_{1}$ and $\boldsymbol{S}_{2}$, and we suggest computing the signed distances $d$ based on Ref. [19] for each point. As distances between surfaces are not symmetrical, i.e. $d\left(\boldsymbol{S}_{1}, \boldsymbol{S}_{2}\right) \neq d\left(\boldsymbol{S}_{2}, \boldsymbol{S}_{1}\right)$, both distances must be computed. Besides, to maintain the coherence of results, the sign of the distances of $d\left(\boldsymbol{S}_{2}, \boldsymbol{S}_{1}\right)$ must be inverted, i.e. multiplied by -1 , because $\boldsymbol{S}_{1}$ is considered the reference surface. The negative and positive signs specify retraction and protrusion of the ACW, respectively.

\subsection{Preliminary study}

We present a preliminary study for investigating if there are changes in the ACW morphology during the initial period after bar removal, and, if so, investigate the potential key factors that might be associated with these changes using statistical analysis.

\subsubsection{Study sample}

Following institutional review board approval, this study was conducted at Centro Hospitalar de São João and Hospital de Braga, in Portugal, from December 2011 to September 2013.

Given the low frequency of PE patients who undergo Nuss procedure, we included the first consecutive patients who met the following criteria: one bar placement and the bar kept in situ for at least two years.
A total of 21 patients were enrolled in this study. Two experienced surgeons performed these surgeries. The bar placement and bar removal were performed according to the Nuss technique and no overcorrection was applied during bar placement.

Table 1 summarizes patients' characteristics and treatment details. The asymmetry was calculated based on the asymmetric index [20] and the correction index as described by St. Peter et al. in Ref. [21]. The sternum's maximum correction was measured as the distance between the sternum's posterior surface and the line representing the bar's real position (Fig. 2-A). Because of the radiation exposure, postsurgical CT scan was not made.

\subsubsection{Acquisition protocol and ACW surfaces assessment}

For each patient, the ACW was acquired at three points:

i) immediately before bar removal;

ii) immediately after bar removal;

iii) 2-8 weeks after surgery. This range was established by the patient's recovery time and the schedule of the follow-up visit.

The evolution of the ACW was studied by overlapping the acquired surfaces at two assessment time points for each patient:

i) Early assessment: Before bar removal $v s$ after bar removal;

ii) Interim assessment: Before bar removal vs 2-8 weeks after bar removal.

To measure the differences in the ACW morphology, one specific anatomical area was considered. This area was restricted by the intermammary line, the two mammillary lines (vertical) and the line close to the transpyloric plane. This area was used to measure variations in the anatomical zone supported by the metal bar (Fig. 2-B).

Concerning this anatomical area, the signed distances (Fig. 2-B) and volumes between the ACW surfaces were computed. Three measurements were estimated:

i) Mean change $(\mathrm{cm})$, the mean distance: $d_{\text {mean }}=\operatorname{mean}\left[d\left(\boldsymbol{S}_{1}, \boldsymbol{S}_{2}\right)\right.$, $\left.d\left(\boldsymbol{S}_{2}, \boldsymbol{S}_{1}\right)\right]$

ii) Maximum change $(\mathrm{cm})$, the largest distance: $d_{\max }=\max \left[d\left(\boldsymbol{S}_{1}\right.\right.$, $\left.\left.\boldsymbol{S}_{2}\right), d\left(\boldsymbol{S}_{2}, \boldsymbol{S}_{1}\right)\right]$

iii) Volume change $\left(\mathrm{cm}^{3}\right)$, estimation of the volume variation: $\mathrm{Vol}=$ $\sum_{i}^{N_{f}}\left(d_{i} \times A_{i}\right)$, where $N_{\boldsymbol{f}}$ represents the total number of triangular faces of $\boldsymbol{S}_{1}, d_{i}$ the closest distance of each triangle to $\boldsymbol{S}_{2}$, and $A_{i}$ the area of each triangle.

To perform the ACW surfaces assessment (i.e. surface alignment, signed distances computation, and measurements at specific anatomical lines and areas), an application in MATLAB ${ }^{\circledR}$ (version R2011b, The Mathworks Inc., Natick, MA) was developed.

\subsubsection{Statistical analysis}

All statistical analyses were conducted using SPSS Statistics (version 21, SPSS Inc., IBM Company, Chicago, IL). The assumption of normality was assessed for all variables involved and, according to the results, parametric or nonparametric tests were applied. The effect size and

Table 1

Patients' characteristics and treatment details $(n=21)$.

\begin{tabular}{ll}
\hline Variable & \\
\hline Haller index & $3.5 \pm 0.5(2.7-4.4)^{\mathrm{a}}$ \\
Correction index (\%) & $29.4 \pm 8.6(6.3-45.1)^{\mathrm{a}}$ \\
Asymmetry (symmetric/asymmetric) & $15 / 6$ \\
Sternum rotation (degrees) & $14 \pm 10(5-45)^{\mathrm{a}}$ \\
Gender (male/female) & $17 / 4$ \\
Age at bar placement (years) & $15 \pm 4(11-29)^{\mathrm{a}}$ \\
Sternum's maximum correction (cm) & $3.2 \pm 0.9(1.7-5.9)^{\mathrm{a}}$ \\
Time the bar was in situ (months) & $33 \pm 4(26-45)^{\mathrm{a}}$ \\
Age at bar removal (years) & $18 \pm 4(13-32)^{\mathrm{a}}$ \\
\hline
\end{tabular}

${ }^{\text {a }}$ Mean \pm standard deviation (min-max). 


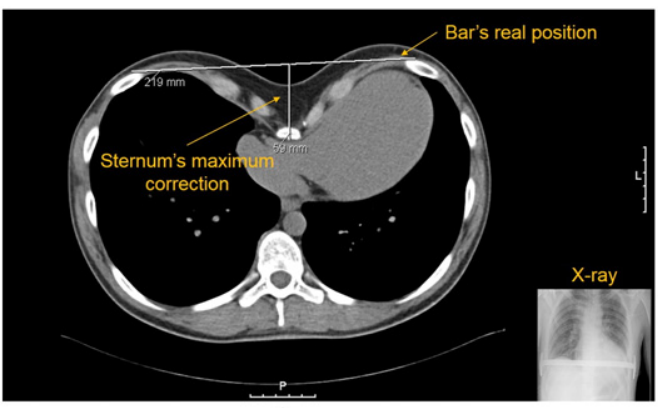

A

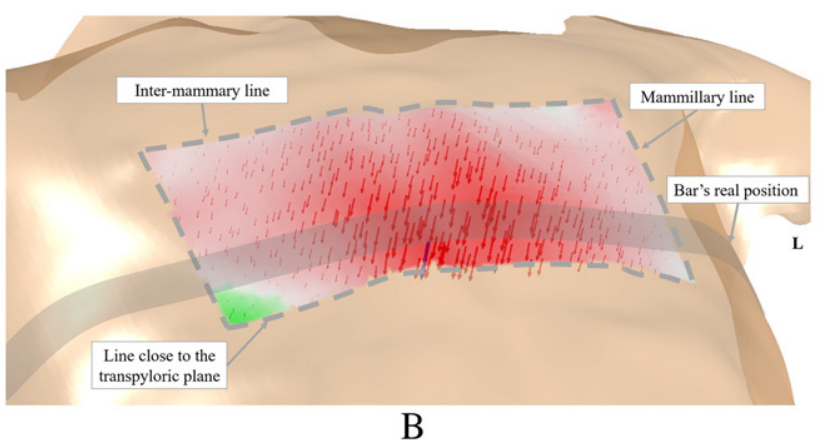

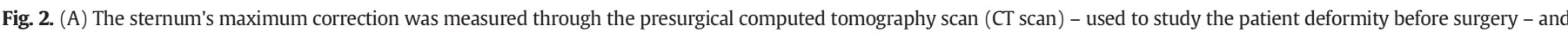

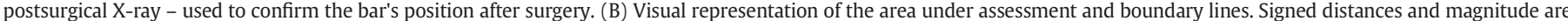

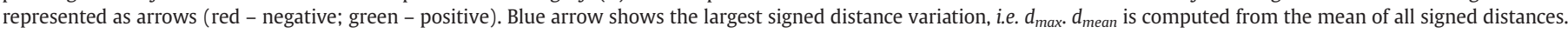
Similarly, the Vol is computed using the signed distances and the area of triangular faces.

statistical significance were reported. All statistics were considered significant if $p<0.05$.

1.2.3.1. Statistical differences between early and interim assessments. The paired sample Student's t-test or Wilcoxon signed rank test was performed to detect if there were differences between the morphological changes that happen immediately and 2-8 weeks after surgery, i.e. between early and interim assessments.

1.2.3.2. Correlation analysis. Correlation analysis was performed to study whether the variability of the ACW changes was dependent on individual characteristics of patients (presented in Table 1). Early assessment, interim assessment, and their paired difference were used. The paired difference between early and interim assessments was included because it represents the amount of retraction that increased at both assessment points. Pearson's correlation (R), point-biserial correlation $\left(R_{p b}\right)$ or Spearman's rho $\left(R_{s}\right)$ was computed according to variable characteristics.

1.2.3.3. Regression analysis. A multiple linear regression (MLR) analysis was conducted to model the relationship between two or more explanatory variables. We intend to understand if multiple explanatory variables can better explain the changes that might occur during assessment points. As previously mentioned, we modeled their relationships using patients' characteristics and treatment details as independent variables (or predictors) and patients' early, interim and paired difference measurements as dependent variables.

All possible subsets of the set of potential independent variables were computed. This approach conducts a computationally intensive search of the entire model space by considering all possible regression models from the pool of potential predictors. The best model was obtained using the minimum value of the Akaike's information criterion corrected $\left(\mathrm{AIC}_{\mathrm{c}}\right.$ ). The $\mathrm{AIC}_{\mathrm{c}}$ was used because it is a more accurate metric for small samples [22,23]. The models were considered statistically significant through the F-statistics. In all models, each predictor was evaluated according to its statistical significance, confidence intervals, unstandardized coefficients, and correlation coefficients.

\section{Results}

\subsection{Early and interim assessments}

Based on the early results (Table 2), the bar removal was accompanied by a slight modification of the ACW shape and a tendency toward retraction was observed (Fig. 3).

Based on the interim results, the tendency observed during the early assessment was also seen for all parameters in the interim (Fig. 3). The Vol, $d_{\text {mean }}$, and $d_{\text {max }}$ measurements increased within the area under evaluation. However, there was no recurrence within our study, i.e. return to the same shape before pectus bar placement, being evaluated by surgeons and patients in the follow-up visit.

The differences and the magnitudes of the differences between early and interim assessments were statistically significant and considerably large based on Cohen's conventions, respectively (Table 2).

\subsection{Correlation analysis}

To perform and present the correlation analysis, we decided to use as dependent variables the results from $d_{\text {mean }}$ measurements. This decision was based on significant correlations of $d_{\text {mean }}$ with $d_{\text {max }}$ and $\mathrm{Vol}$ $(p<0.05)$.

The age at bar placement $\left(\mathrm{R}_{\mathrm{s}}(19)=0.476, p=0.029\right)$ and bar removal $\left(R_{S}(19)=0.466, p=0.033\right)$ was positively, moderately and significantly associated with early retraction. These data suggest that older patients at bar placement and bar removal tended to present less retraction immediately after bar removal surgery.

Similarly, the time the bar was in situ was positively, moderately, and significantly correlated with interim retraction $(\mathrm{R}(19)=0.490$, $p=0.024)$. Thus, longer time with the bar in situ was associated with less retraction in the interim stage. There was a trend for less retraction in the early stage but it did not reach statistical significance $(\mathrm{R}(19)=$ $0.405, p=0.069$ ).

The amount of retraction that occurred between early and interim assessments (paired difference) suggests being lesser for female patients $\left(\mathrm{R}_{\mathrm{pb}}(19)=0.450, p=0.041\right)$. Similarly, this correlation was positive, moderate and significant.

Table 2

Results of early and interim assessments and statistical analysis of the area under evaluation $(\mathrm{n}=21)$

\begin{tabular}{|c|c|c|c|}
\hline & Early $^{\mathrm{a}}$ & Interim $^{\mathrm{a}}$ & Paired sample $t$-test \\
\hline$d_{\text {mean }}(\mathrm{cm})$ & $-0.1 \pm 0.1(-0.5$ to 0.1$)$ & $-0.5 \pm 0.2(-0.8$ to -0.1$)$ & $\mathrm{t}(20)=-8.86 ; p<0.001 ;$ Cohen's $\mathrm{d}=-1.93$ \\
\hline$d_{\max }(\mathrm{cm})$ & $-0.6 \pm 0.2(-1.2$ to -0.3$)$ & $-1.3 \pm 0.3(-2.1$ to -0.7$)$ & $\mathrm{t}(20)=-11.1 ; p<0.001 ;$ Cohen's $\mathrm{d}=-2.42$ \\
\hline $\operatorname{Vol}\left(\mathrm{cm}^{3}\right)$ & $47.8 \pm 22.2(19.4-96)$ & $122.1 \pm 47.3 \mathrm{~cm}^{3}(52.5-239.5)$ & $\mathrm{t}(20)=8.41 ; p<0.001 ;$ Cohen's $\mathrm{d}=1.83$ \\
\hline
\end{tabular}

a Mean \pm standard deviation (min-max). 
A

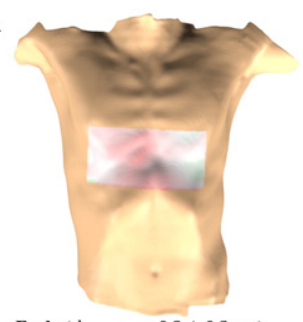

Early $\left(d_{\text {mean }}=-0.3 \pm 0.2 \mathrm{~cm}\right)$

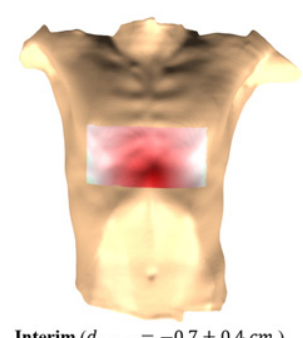

$\operatorname{Interim}\left(d_{\text {mean }}=-0.7 \pm 0.4 \mathrm{~cm}\right)$
B

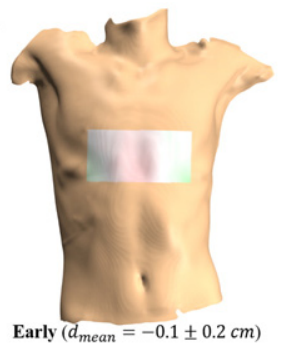

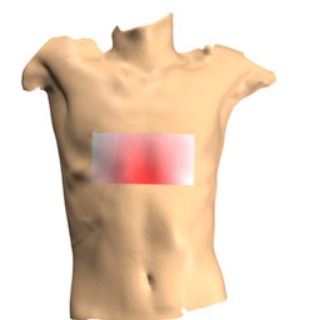

Interim $\left(d_{\text {mean }}=-0.7 \pm 0.4 \mathrm{~cm}\right)$
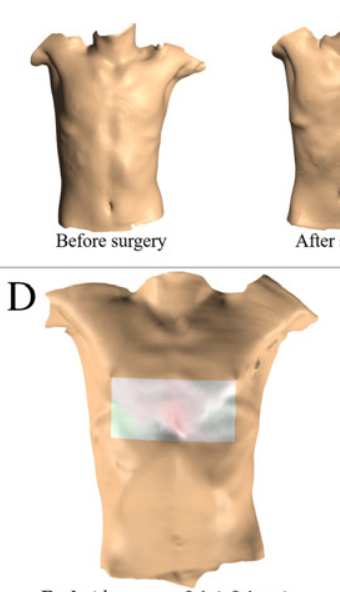

Early $\left(d_{\text {mean }}=-0.1 \pm 0.1 \mathrm{~cm}\right)$
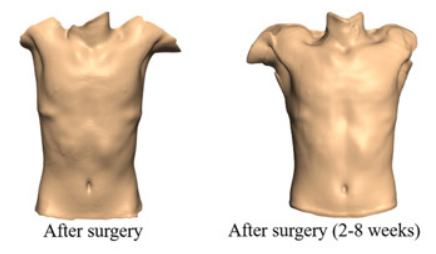

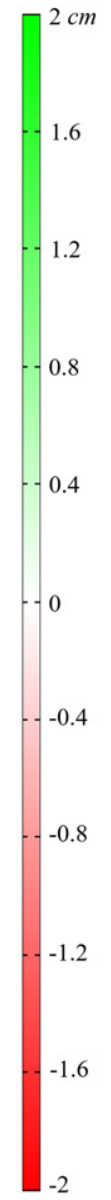

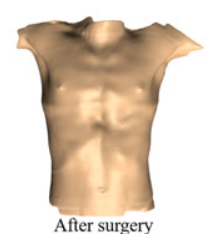

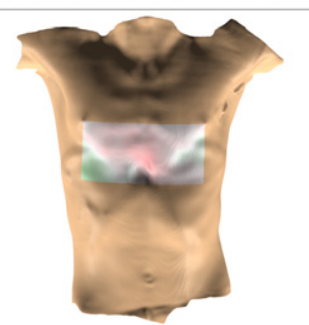

Interim $\left(d_{\text {mean }}=-0.1 \pm 0.2 \mathrm{~cm}\right)$

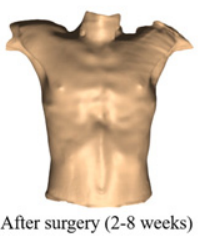

$-1.2$

.6

$-2$

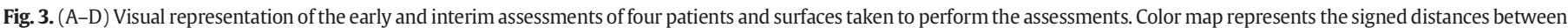
surfaces (red - retraction; green - protrusion).

Reviewing, data suggest that female patients, older patients and patients who kept the bar in situ for a longer period each correlated positively and significantly with less ACW postretraction.

\subsection{Regression analysis}

The $d_{\text {mean }}$ measurement was also used to compute the MLR and, as predictors, we used variables that followed normality assumption. The results are presented in Table 3. There were no factors significantly associated with early-stage retraction.
On the interim stage, the model indicated that the time the bar was in situ was associated with higher and positive effect (partial R (19) = $0.613, p=0.005$ ) on reducing postretraction when the correction index and gender were controlled. This result reinforced the value of the time the bar is in situ in predicting ACW postretraction.

The paired difference model was statistically significant, with gender exposing more significant effect (partial $\mathrm{R}(19)=0.505, p=0.023$ ) when the time the bar was in situ was controlled.

Reviewing the MLR models, which represent the mean retraction of the ACW, data suggest that female patients and patients who kept the

Table 3

Regression models.

\begin{tabular}{|c|c|c|c|c|c|c|c|c|c|c|c|c|}
\hline \multicolumn{7}{|l|}{ Model summary } & \multicolumn{6}{|l|}{ Model coefficients } \\
\hline Dependent variable & $F_{(\mathrm{df1}, \mathrm{df} 2)}$ & $p$ & $\mathrm{R}$ & $\mathrm{R}^{2}$ & $\mathrm{R}^{2}$ adjusted & $\mathrm{AIC}_{\mathrm{c}}$ & $($ Constant $)+$ predictors & $\mathrm{B} \pm \mathrm{SE}$ & [CI 95\%] & $\beta$ & $p$ & Partial R \\
\hline \multirow[t]{2}{*}{ Early } & $3.728_{(1,19)}$ & 0.069 & 0.405 & 0.164 & 0.120 & -87.447 & (Constant) & $-0.534 \pm 0.205$ & {$[-0.963 ;-0.105]$} & & 0.017 & \\
\hline & & & & & & & Time the bar was in situ & $0.012 \pm 0.006$ & {$[-0.001 ; 0.025]$} & 0.405 & 0.069 & 0.405 \\
\hline \multirow[t]{4}{*}{ Interim } & $4.135_{(3,17)}$ & $0.023^{*}$ & 0.650 & 0.422 & 0.320 & -71.928 & (Constant) & $-1.248 \pm 0.285$ & {$[-1.848 ;-0.647]$} & & 0.001 & \\
\hline & & & & & & & Time the bar was in situ & $0.028 \pm 0.009$ & {$[0.009 ; 0.046]$} & 0.618 & $0.005^{* *}$ & 0.613 \\
\hline & & & & & & & Correction index & $-0.007 \pm 0.004$ & {$[-0.016 ; 0.002]$} & -0.315 & 0.120 & -0.369 \\
\hline & & & & & & & Gender & $0.171 \pm 0.090$ & {$[-0.018 ; 0.360]$} & 0.364 & 0.073 & 0.421 \\
\hline \multirow[t]{3}{*}{ Paired difference } & $3.684_{(2,18)}$ & $0.046^{*}$ & 0.539 & 0.290 & 0.212 & -70.102 & (Constant) & $-0.865 \pm 0.306$ & {$[-1.507 ;-0.223]$} & & 0.011 & \\
\hline & & & & & & & Time the bar was in situ & $0.014 \pm 0.009$ & {$[-0.006 ; 0.033]$} & 0.301 & 0.152 & 0.332 \\
\hline & & & & & & & Gender & $0.238 \pm 0.096$ & {$[0.036 ; 0.440]$} & 0.499 & $0.023^{*}$ & 0.505 \\
\hline
\end{tabular}

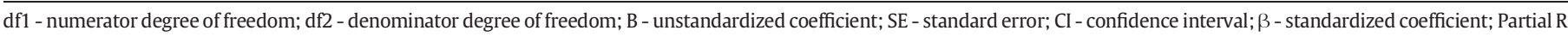
- partial correlation; $\mathrm{AIC}_{\mathrm{c}}$ - Akaike's information criterion corrected.

MLR of the $d_{\text {mean }}$ for early, interim and paired difference. Best models were obtained using all possible subsets.

* $p<0.05$.

** $p<0.01$. 
bar in situ for a longer period each correlated positively and significantly with less ACW postretraction.

\section{Discussion}

PE surgical repair has been increasingly used, mainly because of the good results of the surgical procedures [3,24]. However, based on our clinical experience and reported data, it has been observed that Nuss procedure outcomes following bar removal are not always satisfactory. This is a problem because the process is used to correct a deformity that inflicts psychological and social harm in patients [25]. Therefore, a great deal of progress must be made before perfect surgical results may be obtained.

The success or the failure of the Nuss procedure has been mostly based on the surgeon's subjective evaluation of the results, as well as questionnaires answered either by the patients or by their parents. To objectively understand how the ACW behaves over time, this work presents a novel methodology to assess the ACW modifications following bar removal based on 3D surface data. As well, this work presents a preliminary study using this methodology. To the best of our knowledge, this study is the first using 3D surface data to assess the postsurgical outcome of PE correction after bar removal.

A study, which focused only on the two-dimensional contour under the bar's position, showed that retraction occurs following bar removal [10]. This study measured the differences in the height and the width of the two-dimensional contour obtained from a thermoplastic strip. The differences in the height of ACW may be compared with our interim $d_{\max }$ values. In fact, before and after bar removal, the height differences $(-1.1 \pm 0.6 \mathrm{~cm}, \mathrm{n}=26)$ were similar to our preliminary results. During each of these studies, the bar was kept into the retrosternal position for at least 2 years. Using different methodologies and studying different populations (different genetic background), each of these studies reinforced, proved and quantified the clinical idea of postsurgical retraction. With $3 \mathrm{D}$ assessment, we observed that the volume variation was small in the area under assessment which corresponds to a small variation of the average height of the ACW. Moreover, these variations grew gradually over time where the postsurgical retraction occurred immediately following bar removal and increased in the weeks following surgery. Data suggest some influence on visual perception of ACW retraction. The retraction may be unnoticed by most of the patients and surgeons because of its gradualness and being present in different proportions along the chest surface (Fig. 3). Albeit, as future work, a correlation between visual perception/satisfaction and quantified values of the ACW retraction must be made.

The correlation analysis in the early stage revealed positively, moderately and statistically significant correlations between the age at bar placement and bar removal. This suggests that patients who undergo Nuss procedure in older ages tended to present less retraction immediately after bar removal. This is connected with previous findings, where Nuss observed that the age at the time of repair affects recurrence rate [9]. Equally, the correlation analysis in the interim stage found that the time the bar was in situ is positively, moderately and statistically significant. Indeed, there was an inverse and significant relationship between the time the bar was in situ and the amount of ACW retraction. Similarly, these results are consistent with the semiquantitative assessments presented by Nuss [9]. An inverse relationship between the recurrence rate and the time the bar was in situ was observed for cases in which the bar was removed before 2 years had passed. This inverse relationship appears to hold true for ACW retraction when the bar is removed after 2 years. Although there were no cases of recurrence within our study, the results revealed the persistence of the inverse relationship over time, since less postsurgical retraction occurred when the time the bar was in situ was longer. The gender suggests influencing the amount of postretraction that occurs between early and interim assessments.

During correlation analysis, the influence of different intervals (2-8 weeks) between postoperative assessments was studied and it was found to have no correlation. Assessed more than one year after bar removal, the previous semiquantitative study reported over $95 \%$ of good and excellent outcomes when the bar was kept in situ for more than 19 months [9]. Therefore, both studies may indicate that the ACW changes but eventually stabilizes following bar removal. However, assessing the ACW changes at 6 and 12 months after bar removal must be made, and further studies must be performed to overtake the effect of patient growth (when it is present) on surfaces analysis in longterm assessments. Surfaces with different sizes cannot be directly overlapped and compared, as it is proposed in this methodology. Besides, simple resizing techniques may alter the surface morphology and introduce errors on measurements.

The current sample size must be increased and long-term assessments must be made to completely validate the methodology and reinforce our preliminary findings. So far, this methodology revealed useful for monitoring changes in the ACW, avoiding multiple costs and no radiation-free imaging exams such as CT scan. Moreover, the scanner's portability and easy manipulation make it possible to obtain data in different scenarios, as in the operating room.

\section{Conclusion}

A new methodology to evaluate PE correction after bar removal was presented. This methodology is novel, objective and safe, helping on follow-up of pectus excavatum patients. The preliminary study shows that the results obtained with this methodology are related with the literature, where the ACW retraction occurs and data analysis suggests that the time the bar is in situ, gender and age are relevant in ACW retraction following bar removal. Besides, the time the bar is in situ highlights as the main determinant in the ACW retraction following bar removal. However, further studies should continue to corroborate and reinforce these findings, by increasing the sample size and performing long-term assessments. In the future, more quantitative data of PE correction might be useful to compute more complex mathematical models and use them to predict and control postsurgical retraction for each patient.

\section{Funding}

This work has been funded by FEDER funds, through the Competitiveness Factors Operational Programme (COMPETE), and by National funds, through the Foundation for Science and Technology (FCT), under the scope of the projects PTDC/SAU-BEB/103368/2008 and POCI-01-0145-FEDER-007038; and by the projects NORTE-07-0124FEDER-000017 and NORTE-01-0145-FEDER-000013, supported by the Northern Portugal Regional Operational Programme (NORTE 2020), under the Portugal 2020 Partnership Agreement, through the European Regional Development Fund (FEDER).

J. Gomes-Fonseca was funded by FCT through the State Budget of the Ministry of Education and Science and European Social Fund through the Human Potential Operational Program 2007-2013 NSRF Portugal (QREN Portugal 2007-2013) under the scholarship PD/BDE/113597/ 2015. The funders had no role in study design, data collection and analysis, decision to publish, or preparation of the manuscript.

\section{Acknowledgments}

The authors would like to thank the departments of pediatric surgery of Centro Hospitalar São João and Hospital de Braga.

\section{Appendix A. Methodology step-by-step}

The proposed methodology is designed using the Polhemus FastSCAN Cobra model (Polhemus, Colchester, VT). Nevertheless, different Polhemus FastSCAN models may have slight differences or options. Thus, some steps may undergo minor changes. The main ideas of this methodology can be used with scanners with similar acquisition properties, as long as similar surface information is acquired. The step-bystep description of the proposed methodology follows. 


\section{A.1. Surface acquisition (Polhemus FastSCAN scanner):}

1) Position the patient in dorsal decubitus with the arms away from the thorax and parallel to both transverse and coronal planes. The chest must be undressed;

2) Position the reference/transmitter near the patient, so that the entire anterior chest wall is inside the magnetic tracking volume. Using adhesive tape, one can position the reference/transmitter under the fifth or the sixth rib (rigid structures) to make sure that the magnetic tracking volume is holding the entire chest. Verify if the reference/ transmitter is not positioned within the area under evaluation;

3 ) Set the sensitivity in the wand (usually to value 3 );

4) Keep the wand up at, approximately, $20 \mathrm{~cm}$ from the patient's chest. Visualize if the laser light is completely visible on the FastSCAN software by continuously pressing the trigger in the wand (you must hear just one click). Test this step in the entire chest. If the line is not visible in the software, reduce the room light, move the wand closer to or further away from the chest, or set a new sensitivity.

5) Ask the patient to hold breathing (if not possible, ask to control breathing, without sudden movements) and start the acquisition (you must hear two clicks in the trigger);

6) Perform multiple and continuous sweeps from the shoulders and neck to the lumbar and umbilical regions. Move and adjust the wand position and orientation according to the chest contour. Stop when the entire anterior chest wall is acquired. Correct nonacquired chest portions. The sweeps must overlap each other to enable postprocessing. One can verify the scanning resolution and the tracker range in the Color Mapping option.

7) Repeat steps 5-6 to acquire more than one surface sample.

\section{A.2. Surface postprocessing (Polhemus FastSCAN software):}

1) If necessary, eliminate low-quality sweeps, using the Sweeps List option;

2) If necessary, select and delete points in sweeps that one considers to interfere in the postprocessing (as scattered points, shape of the reference/transmitter, stretcher parts, clothes, etc.), using the Select tool and Delete option;

3) Register (or align) the selected sweeps, using the Register Sweeps tool. This tool corrects small misalignments between sweeps;

4) Repeat step 3 until the register error stabilizes.

5) Using Generate Surface tool, merge the sweeps in one surface through the Basic Surface Processing. Set parameters to reduce noise, preserving the current surface shape.

6) Then, establish the degree of smoothness and resolution of the final surface through the RBF Surface Processing parameters in the Generate Surface tool.

A.2.1. An additional step, when more than one surface sample is available for each time point:

7) Select surfaces $\boldsymbol{M}_{i}$ for $i=1, \ldots, N_{\boldsymbol{M}}$, with $N_{\boldsymbol{M}}$ being the total number of the surfaces. Let $\boldsymbol{M}$ denote a triangular mesh, which is represented by a set of points $\boldsymbol{P}$ (or vertices) and a set of triangular faces $\boldsymbol{F}$. Let $\boldsymbol{P}=\left\{\boldsymbol{p}_{j}\right\}$ for $j=$ $1, \ldots, N_{\boldsymbol{p}}$, with $N_{\boldsymbol{p}}$ being the total number of points, and $\boldsymbol{p}=(x, y, z)$ representing the coordinates in the 3D Euclidean space; and let $\boldsymbol{F}=$ $\left\{\boldsymbol{f}_{k}\right\}$ where $\boldsymbol{f}=(a, b, c)$ for $a, b, c \in\left\{1, \ldots, N_{\boldsymbol{p}}\right\}$ indicating the index of each point in $\boldsymbol{P}$ and $k=\left\{1, \ldots, N_{f}\right\}$ where $N_{\boldsymbol{f}}$ is the total number of faces.

8) Merge each surface into a new surface $\boldsymbol{W}$, where $\boldsymbol{W} \supset\left\{\boldsymbol{M}_{1}, \ldots, \boldsymbol{M}_{N_{\boldsymbol{M}}}\right\}$.

9) Reconstruct into a new single surface $\boldsymbol{S}$. Let $\boldsymbol{S}=r(\boldsymbol{W} ; \boldsymbol{\nu})$ where $r$ is the reconstruction method $^{2}$ and $\boldsymbol{\nu}$ the input parameters.

\footnotetext{
2 During this methodology, the method used for surface reconstruction was the screened Poisson surface reconstruction. For mathematical details, consult Ref. [14], and for implementation details, consult http://www.cs.jhu.edu/ misha/Code/PoissonRecon/. The proposed methodology is not restricted to this surface reconstruction method, being possible to use other available methods.
}

A.3. Surfaces assessment - registration/alignment:

1) Select two surfaces, $\boldsymbol{S}_{1}$ and $\boldsymbol{S}_{2}$, at two different time points.

2) Select two corresponding set of landmark points, $\boldsymbol{L}_{1}$ and $\boldsymbol{L}_{2}$, on both surfaces. Let $\boldsymbol{L}_{m} \subset \boldsymbol{S}_{\mathrm{m}}$ and $m=\{1,2\}$. Let $\boldsymbol{L}=\left\{\boldsymbol{l}_{m, n}\right\}$ for $n=\left\{1, \ldots, N_{\boldsymbol{l}}\right\}$, $N_{\boldsymbol{l}}>2$ where $N_{\boldsymbol{l}}$ is the total number of landmark points. For example, select the landmark points on nipples and umbilicus.

3) Register $\boldsymbol{S}_{1}$ to $\boldsymbol{S}_{2}$ :

a. Compute a rigid transformation (rotation $\boldsymbol{R}$ and translation $\boldsymbol{t}$ ) that initially maps $\boldsymbol{L}_{2}$ to $\boldsymbol{L}_{1}$ :

i. For both set of landmark points, $\boldsymbol{L}_{1}$ and $\boldsymbol{L}_{2}$, find the centroid, $\boldsymbol{c}_{1}$ and $c_{2}$ :

$$
\begin{gathered}
\boldsymbol{c}_{1}=\frac{1}{N_{\boldsymbol{l}}} \sum_{n=1}^{N_{\boldsymbol{l}}} \boldsymbol{l}_{1, n} \\
\boldsymbol{c}_{2}=\frac{1}{N_{\boldsymbol{l}}} \sum_{n=1}^{N_{\boldsymbol{l}}} \boldsymbol{l}_{2, n}
\end{gathered}
$$

ii. Recenter both set of landmark points so that the centroids are at the origin, and compute the matrix $\boldsymbol{H}$ :

$\boldsymbol{H}=\sum_{n=1}^{N_{1}}\left(\boldsymbol{l}_{2, n}-\boldsymbol{c}_{2}\right)\left(\boldsymbol{l}_{1, n}-\boldsymbol{c}_{1}\right)^{T}$

where $T$ means the transpose.

iii. Use singular value decomposition (SVD) ${ }^{3}$ algorithm to find the rotation $\boldsymbol{R}$ :

$[\boldsymbol{U}, \boldsymbol{S}, \boldsymbol{V}]=\operatorname{SVD}(\boldsymbol{H})$

$\boldsymbol{R}=\boldsymbol{V} \boldsymbol{U}^{T}$

iv. Find the translation $\boldsymbol{t}$ :

$\boldsymbol{t}=\boldsymbol{c}_{1}-\boldsymbol{R} \times \boldsymbol{c}_{2}$

b. Apply the rigid transformation found in the previous step to the set of points of $\boldsymbol{S}_{2}$;

c. Define the main parameters of the pattern search ${ }^{4}$ method, $\psi$ :

i. Define the objective function $\phi$ of the method, as the iterative closest point (ICP) method which performs a rigid registration between $\boldsymbol{S}_{1}$ and a subset of points of $\boldsymbol{S}_{2}$ surrounding landmarks $\boldsymbol{L}_{2}$. Thus, $\phi$ must:

\footnotetext{
${ }^{3}$ For further mathematical details, consult Ref. [15], and for implementation details, consult https://www.mathworks.com/help/matlab/ref/svd.html.

${ }^{4}$ For further mathematical and implementation details, consult https://www. mathworks.com/help/gads/patternsearch.html.
} 
1. Create the bounding boxes $\boldsymbol{B}_{n}$ surrounding the $\boldsymbol{L}_{2}$ with size $\boldsymbol{\mu}=\left(\mu_{x}, \mu_{y}, \mu_{z}\right)$, where $\mu_{x}, \mu_{y}, \mu_{z} \in \mathbb{R}$ indicates the sizes in the 3D Euclidean space;

2. Select the set of points $\boldsymbol{P}_{\boldsymbol{B}_{n}}$ within $\boldsymbol{B}_{n}$ in the surface $\boldsymbol{S}_{2}$;

3. Create a new surface $\boldsymbol{Q}$ with the set of points $\boldsymbol{P}_{\mathbf{Q}}=$

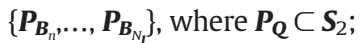

4. Use ICP method to minimize the distance between points of $\boldsymbol{S}_{1}$ and $\boldsymbol{Q}$. Each iteration of this method performs three main steps:

a) For all points $\boldsymbol{q}_{h} \in \mathbf{Q}$ find the closest point $\boldsymbol{g}_{h} \in \boldsymbol{S}_{1}$;

b) Find the rigid transformation that minimizes the squared distance between the transformed $\boldsymbol{q}_{h}$ and the $\mathbf{g}_{h}$ :

$\underset{\boldsymbol{R}, \boldsymbol{t}}{\arg \min } \sum_{h=1}^{N_{q}}\left\|\left(\boldsymbol{R} \boldsymbol{q}_{h}+\boldsymbol{t}\right)-\mathbf{g}_{h}\right\|^{2}$

where ||$. . .||$ is the Euclidean distance between points. The rotation and translation can be computed using the SVD algorithm (previously explained) or a quaternion-based algorithm [16], among others.

c) Apply the estimated rigid transformation to $\mathbf{Q}$ and verify the resulting registration error $e$.

d) If convergence has not been reached, go to step a). Measures of convergence can include the reaching of a minimum registration error, the fact that the closest point pairings in step a) are unchanged, the number of maximum iterations is reached, among others. By repeating the abovementioned steps, the method iteratively optimizes the applied rigid transformation by progressively reducing the registration error $e$ between points.

ii. Then, set the remaining pattern search parameters: the maximum number of iterations $N_{I}$ for the search; the tolerance error $\epsilon$ for delta $\Delta(e)$ to stop the search; the starting point $\boldsymbol{\mu}_{0}$ of the bounding box sizes surrounding each landmark point of $\boldsymbol{L}_{2}$; and the lower $\boldsymbol{\mu}_{\min }$ and upper $\boldsymbol{\mu}_{\max }$ limits of the search for each bounding box.

d. Find the best fitting between $\boldsymbol{S}_{1}$ and the best subset of points of $\boldsymbol{S}_{2}$ that represents the minimum $e$ of all subset of points submitted to the search. For this, optimize $\psi\left(\phi\left(\boldsymbol{S}_{1} ; \boldsymbol{Q} ; \boldsymbol{\mu}\right) ; \boldsymbol{\mu}_{0} ; \boldsymbol{\mu}_{\text {min }} ; \boldsymbol{\mu}_{\text {max }} ; \epsilon ; N_{I}\right)$, where per iteration, $\boldsymbol{\mu}$ is changed and converged to the best final rigid transformation.

e. Apply the final rigid transformation found, based on this second alignment step, to the entire set of points of $\boldsymbol{S}_{2}$.

\section{A.4. Surfaces assessment - distances:}

1) Compute the signed distances ${ }^{5}$ between $\boldsymbol{S}_{1}$ and $\boldsymbol{S}_{2}$, forward $d\left(\boldsymbol{S}_{1}, \boldsymbol{S}_{2}\right)$ and backward $d\left(\boldsymbol{S}_{2}, \boldsymbol{S}_{1}\right)$, for each set of points. Thus, for $d\left(\boldsymbol{S}_{1}, \boldsymbol{S}_{2}\right)$ :

a. Let there be a point $p \in \boldsymbol{S}_{1}$, and assume that point $\boldsymbol{c}$ is the closest point in $\boldsymbol{S}_{2}$ for $\boldsymbol{p}$, so that distance $d=\|\boldsymbol{p}-\boldsymbol{c}\|$. Let $\boldsymbol{n}_{\alpha}$ be the sum of normal to faces $\boldsymbol{n}_{k}$ incident on $\boldsymbol{p}$, weighted by the angle $\alpha_{k}$ of the incident face, i.e.:

$\boldsymbol{n}_{\alpha}=\sum_{k} \boldsymbol{n}_{k} \alpha_{k}$

\footnotetext{
${ }^{5}$ For further mathematical details, consult Ref. [19].
}

b. Consider the vector $\boldsymbol{u}=\boldsymbol{p}-\boldsymbol{c}$.. For $D=\boldsymbol{n}_{\alpha} \cdot \boldsymbol{u}$, it holds that $D>0$ if $\boldsymbol{c}$ is outside the surface, and $D<0$ if $\boldsymbol{c}$ is inside. Thus, the signed distance for each point $\boldsymbol{p}$ can be given by:

$d=\|\boldsymbol{u}\| \operatorname{sign}\left(\boldsymbol{u} \cdot \boldsymbol{n}_{\alpha}\right)$

c. Compute the signed distances $d$ for the entire set of points.

d. For backward distance $d\left(\boldsymbol{S}_{2}, \boldsymbol{S}_{1}\right)$, invert the signs to keep the same sign direction of $d\left(\boldsymbol{S}_{1}, \boldsymbol{S}_{2}\right)$.

2) Using landmark points, establish the area under evaluation. For example, select the nipples and a point close to transpyloric plane.

3) Define a bounding box using the landmark points and, based on the signed distances within the bounding box, compute some metrics as:

a. Mean distance, $d_{\text {mean }}=\frac{1}{N_{p}} \sum_{i=1}^{N_{p}} d_{i}$;

b. Maximum distance, $d_{\max }=\max _{i \in N_{p}}\left(d_{i}\right)$;

c. Volume, $\mathrm{Vol}=\sum_{i=1}^{N_{f}}\left(d_{i} \times A_{i}\right)$.

with $N_{\boldsymbol{p}}$ being the total number of points, $N_{\boldsymbol{f}}$ the total number of triangular faces inside the bounding box, and $A_{i}$ the area of each triangular face.

\section{References}

[1] Nuss D, Kelly RE. Indications and technique of Nuss procedure for pectus excavatum. Thorac Surg Clin 2010;20:583-97. http://dx.doi.org/10.1016/j.thorsurg.2010.07.002.

[2] Fokin AA, Steuerwald NM, Ahrens WA, et al. Anatomical, histologic, and genetic characteristics of congenital chest wall deformities. Semin Thorac Cardiovasc Surg 2009;21:44-57. http://dx.doi.org/10.1053/j.semtcvs.2009.03.001.

[3] Johnson WR, Fedor D, Singhal S. Systematic review of surgical treatment techniques for adult and pediatric patients with pectus excavatum. J Cardiothorac Surg 2014;9: 25. http://dx.doi.org/10.1186/1749-8090-9-25.

[4] Nuss D, Kelly Jr RE, Croitoru DP, et al. A 10-year review of a minimally invasive technique for the correction of pectus excavatum. J Pediatr Surg 1998;33:545-52. http:// dx.doi.org/10.1016/S0022-3468(98)90314-1.

[5] Kelly RE, Cash TF, Shamberger RC, et al. Surgical repair of pectus excavatum markedly improves body image and perceived ability for physical activity: multicenter study. Pediatrics 2008;122:1218-22. http://dx.doi.org/10.1542/peds.2007-2723.

[6] Hanna WC, Ko MA, Blitz M, et al. Thoracoscopic Nuss procedure for young adults with pectus excavatum: excellent midterm results and patient satisfaction. Ann Thorac Surg 2013;96:1033-8.

[7] Kelly RE, Goretsky MJ, Obermeyer R, et al. Twenty-one years of experience with minimally invasive repair of pectus excavatum by the Nuss procedure in 1215 patients. Ann Surg 2010;252:1072-81. http://dx.doi.org/10.1097/SLA.0b013e3181effdce.

[8] Ishimaru T, Kitano Y, Uchida H, et al. Growth spurt-related recurrence after Nuss procedure. J Pediatr Surg 2009;44:e13-6. http://dx.doi.org/10.1016/j.jpedsurg.2009.04.014.

[9] Nuss D. Minimally invasive surgical repair of pectus excavatum. Semin Pediatr Surg 2008;17:209-17. http://dx.doi.org/10.1053/j.sempedsurg.2008.03.003.

[10] Chang P-Y, Chang C-H, Lai J-Y, et al. Analysis of changes to the anterior chest wall after the Nuss procedure - an objective measurement of pectus excavatum. J Pediatr Surg 2009;44:2291-5. http://dx.doi.org/10.1016/j.jpedsurg.2009.07.053.

[11] Poncet P, Kravarusic D, Richart T, et al. Clinical impact of optical imaging with 3-D reconstruction of torso topography in common anterior chest wall anomalies. J Pediatr Surg 2007;42:898-903. http://dx.doi.org/10.1016/j.jpedsurg.2006.12.070.

[12] Perez JM, Schreiner S, Gorton GE. Evaluation of the VITUS smart laser scanner for accuracy, resolution and repeatability for clinical assessment of pectus deformities and scoliosis. Proc. IEEE 32nd Annu. Northeast Bioeng. Conf., IEEE; 2006. p. 33-4. http:// dx.doi.org/10.1109/NEBC.2006.1629738.

[13] Moreira AHJ, Fonseca JG, Rodrigues PL, et al. Assessment of 3D scanners for modeling pectus carinatum corrective bar. In: Battiato S, Braz J, editors. VISAPP 2013- proc. int. Conf. Comput. Vis. Theory Appl., Barcelona; 2013. p. 122-5.

[14] Kazhdan M, Hoppe H. Screened Poisson surface reconstruction. ACM Trans Graph 2013;32:1-13. http://dx.doi.org/10.1145/2487228.2487237.

[15] Eggert DW, Lorusso A, Fisher RB. Estimating 3-D rigid body transformations: a comparison of four major algorithms. Mach Vis Appl 1997;9:272-90. http://dx.doi.org/ 10.1007/s001380050048.

[16] Besl PJ, Mckay HD. A method for registration of 3-D shapes. IEEE Trans Pattern Anal Mach Intell 1992;14:239-56. http://dx.doi.org/10.1109/34.121791.

[17] Audet C, Dennis JE. Analysis of generalized pattern searches. SIAM J Optim 2002;13: 889-903. http://dx.doi.org/10.1137/S1052623400378742.

[18] Lewis RM, Shepherd A, Torczon V. Implementing generating set search methods for linearly constrained minimization. SIAM J Sci Comput 2007;29:2507-30. http://dx. doi.org/10.1137/050635432. 
[19] Bærentzen JA, Aanæs H. Signed distance computation using the angle weighted pseudonormal. IEEE Trans Vis Comput Graph 2005;11:243-53. http://dx.doi.org/ 10.1109/TVCG.2005.49.

[20] Kilda A, Basevicius A, Barauskas V, et al. Radiological assessment of children with pectus excavatum. Indian J Pediatr 2007:74:143-7.

[21] St. Peter SD, Juang D, Garey CL, et al. A novel measure for pectus excavatum: the correction index. J Pediatr Surg 2011;46:2270-3. http://dx.doi.org/10.1016/j.jpedsurg.2011.09.009.

[22] Hurvich CM, Tsai C-LL. Regression and time series model selection in small samples. Biometrika 1989;76:297-307. http://dx.doi.org/10.1093/biomet/76.2.297.
[23] Burnham KP. Multimodel inference: understanding AIC and BIC in model selection. Sociol Methods Res 2004;33:261-304. http://dx.doi.org/10.1177/ 0049124104268644.

[24] Nasr A, Fecteau A, Wales PW. Comparison of the Nuss and the Ravitch procedure for pectus excavatum repair: a meta-analysis. J Pediatr Surg 2010;45:880-6. http://dx. doi.org/10.1016/j.jpedsurg.2010.02.012.

[25] Habelt S, Korn S, Berger A, et al. Psychological distress in patients with pectus excavatum as an indication for therapy. Int J Clin Med 2011;2:295-300. http://dx. doi.org/10.4236/ijcm.2011.23050. 